\title{
A Research on Architecture of Digital Publishing Management System
}

\author{
Yu Tian \& Jingliang Chen \\ Business School, University of Shanghai for Science and Technology, Shanghai 200093, China
}

Tel: 86-21-5527-5843Ｅ-mail:mr.tianyu@gmail.com

\begin{abstract}
With the development of digital technology, while digital publishing provide convenience for people to access to information it also influences the management mode of traditional copyrights and challenges current copyrights system. In this context, how to protect digital copyrights and promote the healthy development of digital publishing industry through the creation, management, protection and application of copyrights have become the common concerns to the fields of management, intellectual property and digital publishing industry.
\end{abstract}

Keywords: Digital publishing, Copyright, DRM technology, Management system

Currently the world publishing industry is facing a new technology revolution characterized by digitization. Digital publishing has permeated into every stage of publishing industry from the update of medium and process reengineering to the management of contents and the changes of reading methods and altered the means of production and concept of consumption of traditional publishing, which represents the trend of development and direction of press industry in $21^{\text {st }}$ century. Digital publishing technology, digital publishing printing, duplication and developing new media have been included in the focus points of national Science \&Technology Innovation. Developing new cultural industry characterized by digital contents, digital production and network communication rapidly so as to realize the transformation from traditional publishing industry to digitalization has been a national strategy.

\section{The copyrights protection in the environment of digital publishing is urgent.}

The copyrights protection in the environment of digital publishing has become a common concern in the field of intellectual property protection over the world at present. There are some characteristics for digital publishing in the context of network communication as follows:

1) The rapid increasing of kinds and numbers of copyright work, the continuous expanding of creation, dissemination and usage of copyright work and the application of digital technology and the popularity of the Internet make more people participate in the creation and disseminate their works to the public voluntarily. It is possible for each internet user to become the creator, disseminator and user of copyright works.

2) The new problems of copyright protection in the environment of digital publishing appeared unceasingly and the contradiction is becoming more and more outstanding. The digital technique of network is moving forward everyday and the way to use internet is also constantly changing. The digital copyright protection is also facing new problems constantly.

3) Due to the development of digital technology and in network, it is increasingly difficult to protect copyrights. With the increase of network bandwidth and the improvement of quality of transmission, it is easier to copy, disseminate and use others' works. Everyone will be accessed to the works as long as he clicks the mouse and then disseminates them out once again soon. Therefore, digital works is subjected to more harm than traditional works.

4) Some websites duplicate illegally, upload and disseminate other people's works in large amount without authorization and engage in illegal business of digital products such as online game in the forms of "private server", "outside hang" and so on, which not only infracts the legal interest of owners seriously and disturbs the normal orders of operation and dissemination of digital publication so as to influences the healthy development of digital publishing industry, but also brings shocks to traditional industries such as book, music, film and television and so on.

The development of digital publishing industry requires the innovation of products and contents which must be guaranteed by perfect digital publishing management system. Only the relationship between the authorization and use of traditional works under internet environment is handled appropriately, the coordinated development of digital publishing and traditional publishing, music and film and television industries will be realized. Therefore, whether the digital publishing management system is further constructed so as to protect digital copyrights effectively and crack down on the deeds such as piracy becomes the key to the healthy development of digital publishing industry. 


\section{The current status of digital copyrights technology}

\subsection{The application of DRM}

The characteristics of digital information decide there must be a unique technology to strength the protection for the copyrights of digital publication, which is the digital right management(DRM). The working principles of DRM are as follows: firstly establishing a rights issuer of digital publishing. The contents of digital publishing after code compressing may be locked using key. The Key ID and URL of the rights issuer are placed on the top of the locked digital contents. When the users request they may pass the authentication and authorization of the rights issuer of digital contents and unlock according to the Key ID and URL information on the top of program and then the content will be played. The contents needed to be protected are locked so they could not be used without authentication and authorization even though the users download them, which protects strictly the copyrights of contents consequently.

From this we can see that each stage such as the production, circulation and demonstration etc. of digital publishing can not do without DRM technology. The core of DRM technology is to protect the benefit of digital publishers and authors so as to ensure that the digital contents can not be copied and duplicated arbitrarily without authorization, meanwhile, they are not been tampered with. What's more, DEM technology ensures the transaction of digital contents can be counted so that the retransmission of digital contents becomes controllable. Of course to further promote the healthy development of digital publishing industry requires not only perfect DRM technology system but also supports from many kinds of aspects such as policies, law, management and so.

\subsection{The diversification of digital publishing service forms}

The second layer of the current status of digital publishing is the circulation of digital contents and their service forms have become diversification, in which the most common are the sales ways facing to the customer(B2C) including readers can buy electronic books, read on the website, electronic news, listen to digital music, watch digital video etc, besides digital publishing service facing to the library.

The second ways are in accordance with the service ways of this business or the ways of subscription. For example electronic books can be sold by copies; electronic news and electronic journals can be sold by pieces and the websites such as Newstand, Zinio etc. provide the sales way for electronic news or journals abroad; Of course they also provide subscription for electronic news or journals monthly or annually.

The third service way is to provide data retrieval and readings for readers in the form of database, which has been used for many years in libraries abroad. The service form of database is very suitable for resources services such as reference books and instruction and some publishers also provide database home, for example, China encyclopedia publishing house provides website and Commercial Press provide online reference books.

The other service form of digital contents is comprehensive service website which integrates the functions such as full-text retrieval, browse, shopping guidelines and borrowing guidelines in order to provide more overall services.

\subsection{The diversification of digital publishing contents presentation forms}

The reading as the original was is still the main stream of digital publishing at this stage which is adopted by most electronic books, electronic news and electronic journal. Secondly digital publishing is presented in the form of database, for example, the website Lexisnexis, which is a typical example and provide all kinds of resource about law so as to provide teaching and retrieval services for students and lawyers. Furthermore, there is another presentation form combining websites, multi-media and the original versions. Take the website of digital newspaper in Shanghai Xinmin Evening News as an example. Users not only read directly on the website but also download the original articles and digital newspaper by multi-media to read. Another presentation technology is mobile reading. After recent years of development, hand-held devices technology become mature gradually and the technology reading in the mobile phone becomes more and more practical.

\subsection{The current status of the production and making technology of digital publishing}

Now in the newspaper offices, the production and making of digital publishing have been very advanced and the digital assets management technology has been put into practice. Digital asset management system not only manages all kinds of digital assets including newspaper layout, articles, multi-media information, advertisement and release information etc., but manages the digital assets collecting, processing, realize, reuse and their operation. With the digital assets management system it is easy to make digital newspaper.

But the situations are different in publishing houses where digital assets management technology is relatively 
backward. Although parts of publishing houses such as Encyclopedia of China Publishing House applied the technology long ago, most of the rest lack the management of data and digital assets and the digital assets management system so that the layout files were poorly kept and the situations often happen such as files not found, image files lacked or premium characters files lacked and it is difficult to reuse the layout files. Another problem is, without the index of metadata in the production and making of books many links such as bookshops, libraries etc. in the field of circulation of books are making metadata causing much repetition work. Therefore, confronted with the development of digital publishing, the establishment of digital publishing management system based on copyrights protection should be placed on the agenda urgently.

\section{The architecture of digital publishing management system}

\subsection{The development trend of DRM technology}

Many people who bought electronic books feel the operation of purchasing process is complex so it is important to improve the usability of DRM for promoting the development of digital publishing industry. For example, the ways such as the realization of auto registration and the plug and play of hand-hold reading devices reduce the operation for readers. Now academia are discussing the issue of overprotection and studying the flexible mechanism to prevent overprotection. For example, provide the mechanism that the publisher set the words limitation for copying and printing aiming to each book. For another example, developing the technology of partial sharing makes every reader can read on the limited equipments such as PC computer, mobile phone or hand-hold device after they buy a electronic book but there is restriction to for others to copy. In addition, to improve the security of DRM, we also have to study the protection mechanism of DRM system by itself including protocol update and system auto-update etc.. With the increase and popularity of mobile reading we need to solve the techniques of DRM cross-platform hardware adaptability and combining encryption with digital watermarking in order to adapt to more digital publishing service modes.

\subsection{The advantages of digital publishing management system}

The rapid development of digital publishing management system based on copyrights protection adapts to the tide of digital publishing industry and shows very strong competitive advantages. Digital publishing management system can realize deep service of contents through digital assets management so that the contents of management become smaller in size which not only manages the data of the full book but manages the chapters and clause in each book so as to realize the re-processing of contents in order that the reprint and the service of database become more convenient in the future.

Digital publishing management system also can realize personalized service. For example reset the contents of digital book according to different readers. Through combining with lots of digital media information, the platform of digital publishing management system can realize lots of publication forms such as one-time production and release or automatic layout including printed books, electronic books, database, webpage interactivity and mobile phone books etc.

To realize digital publishing management system the important is to make structured information exist in each link of the production, publication and release of the contents. The structured information includes digital metadata information such as the title of the book and the authors, the structure and chapters of the book and the literature citation information. With them we will save a lot of manual input in the stage of circulation of digital publishing and the reuse and re-processing of digital contents become more convenient.

\subsection{The architecture of digital publishing management system}

Digital publishing management system is established on the basis of technique related to a series of behaviors including the description, identification, transaction, protection, monitoring and tracking etc. and the key includes the techniques of digital watermarking, digital fingerprint, right expression language, secure containment and user identification and authentication. After comparing and evaluating the adaptability and performances of all kinds of techniques in internet and mobile network(or wireless network), according to the requirements of digital copyrights management in internet and mobile network we put forward that digital publishing management system consists of four main subsystem as follows:

1) the production system of digital contents includes: the editing and typesetting, processing, the review process etc.

2) the resource management platform of digital publishing manages overall contents including digital media, figures, characters and articles etc.

3) the support platform for compound publishing production based on the resource management platform of 
digital publishing creates digital contents to printed traditional books, electronic books and database etc. through the subsystem such as automatic typesetting and compound DRM etc.

4) the top service platform of digital publishing production provides the services such as release and sales etc. for the products in forms of electronic books and databases etc.

\section{Conclusions}

The digital publishing management system has done well in the aspect of copyright protection, but it also has large vacancy in the aspect of popularizing users and faces some challenges. When the exterior legal users visit the information protected by DRM, they must remount to the policy server of DRM, which means that the policy server will be exposed on the Internet, so it may bring to hidden dangers. So one "gateway server" has to be built around the network periphery and the server permits the customs distinguish and obtain the passwords in the situation of the main policy server isolating from the Internet. Introducing the exterior users also means that the exterior client machines have to be joint into the lists and allocated the account numbers. Once the exterior customs are added, the safety may become complex, but which is not impossible. If a large company supports to register the exterior users through the digital publishing management system, it can expand the purview management to the business associates.

Secondly, how to make the employee be used to the work flow of digital publishing management system? Users may hate the software which disturbs the old working habits. However, any system which complicates visiting data may lead the employee to give up it. So the solution of searching a digital publishing management system being transparent for the users can widen the usage of the system.

Finally, the products of digital publishing management system need a good mechanism of data backup and disaster recovery. Regardless of which solution being adopted, it all needs the main server to control the file purview. The main server going wrong means that the users may isolate from the key business data. The digital publishing management system needs a good mechanism of data backup and disaster recovery, which means that if the enciphered data has not been estimated about the disaster recovery, it may be the final reason accounting for the disappearance of the software of digital publishing management system.

Besides the factors of safety, there are also some other factors to be concerned when the digital publishing management system is extended. Now this kind of software is expensive, which commonly makes use of the node numbers of inner network configuration as the accounting unit; how the software of digital publishing management system can resolve the problem of contacting the external users, for example, one file is encrypted interiorly, but it can not been encrypted when it is sent to the customs; how to resolve the problem of reading purview of files et al.

In conclusion, although many problems are in existence, the products of digital publishing management system can be improved gradually driven by the need of digital publishing customs and digital publishing enterprises.

\section{References}

Cai, Weihong \& Deng, Yuqiao. (2006). Digital rights management system with equity anonymity. Journal of Computer Applications. 2006,26(12):2924-2927.

Chen, Lei, Sha, Ying, Wang, Xiaodong, Zhong, Shangping. (2006). Design and Implementation of DRM based P2P system. Computer Engineering and Applications. 2006(S1):113-116.

Ma, Jin, Zhou, Anmin, Wu, Shaohua, Liu, Ci. (2007). Research on A IPTV Rights Protected Model Based on DRM Technology. MICROCOMPUTER INFORMATION. 2007,23(24):34-36.

Wang, Lingyan, Xu, Zhiqiang. (2006). An Adaptive Pricing and Timing Mechanism for Digital Rights Management. New Technology of Library and Information Service. 2006(11):41-45.

Xiao, Shangqin, Lu, Zhengding, Ling, Hefei, Zou, Fuhao. (2007). A Trust-Scheme-Based DRM Model for P2P System. Journal of Computer Research and Development. 2007, 44(4): 567-573.

$\mathrm{Xu}$, Haiyin, She, Dangen, Li, Dan \& Dong, Jiushan. (2007). Research on DRM system architecture and protocol based on P2P. Computer Engineering. 33(12):122-124.

Xu, Xinguang. (2006). Technology and Problem of DRM. Radio \& TV Broadcast Engineering. 2006(12):75-79.

Yang, Haisheng \& Wu, Yangfan. (2006). Study and Implementation of Digital Rights Management Technology. Radio \& TV Broadcast Engineering. 2006(11):62-66.

Zhang, Jianming, Wen, Xuejun. (2004). Principle and Application on Digital Right Management System. New Technology of Library and Information Service. 2004(02):13-17. 


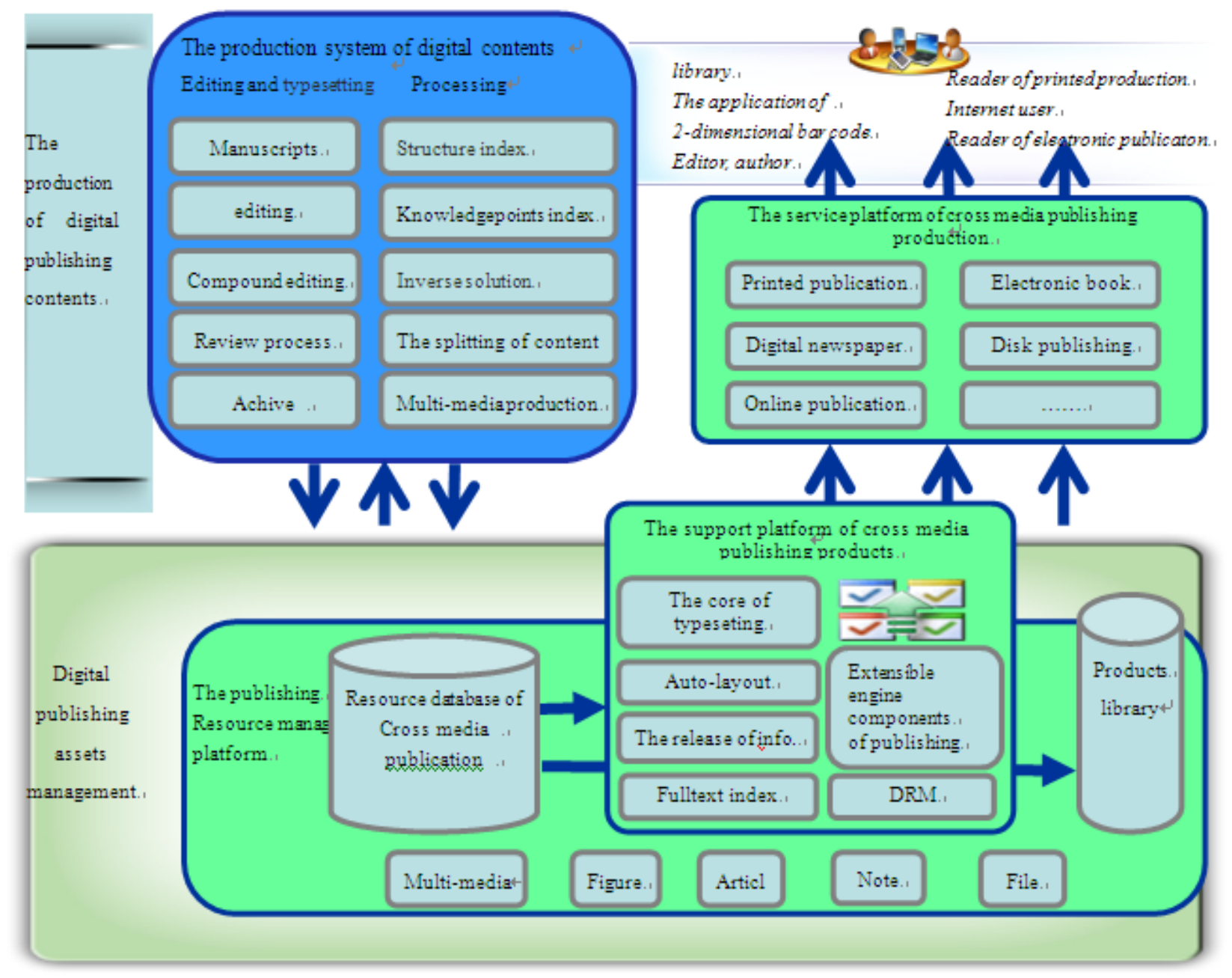

Figure 1. The Architecture of Digital Publishing Management System 